\title{
Specific interaction of plant HMG-like proteins with cruciform DNA
}

\author{
Marcella Bracale ${ }^{1}$, Maria Grazia Galli ${ }^{1,3}$, Christian Savini ${ }^{1}$ and Marco E. Bianchi ${ }^{2}$ \\ ${ }^{1}$ CNR and Dipartimento di Biologia, Sezione Botanica Generale, via Celoria 26, I-21033 Milano, Italy \\ ${ }^{2}$ Dipartimento di Genetica e Biologia dei Microorganismi, via Celoria 26, I-20133 Milano, Italy
}

Received 29 April 1994; Accepted 22 June 1994

\begin{abstract}
Proteins which, on the basis of their solubility in $0.35 \%$ $\mathrm{NaCl}-2 \%$ TCA and of their electrophoretic mobility, correspond to animal HMG $1 / 2$ family were isolated from nuclei of ungerminated pea embryos. These proteins ound with a high degree of specificity to synthetic cruciform DNA produced by annealing chemically synthesized oligonucleotides. Hence, specific binding to four-way junction DNA, prevlously reported for animal HMG 1 and 2 proteins proved also to be a property of plant HMG $1 / 2$ family, in spite of their low homology to the animal ones.
\end{abstract}

Key words: Pisum sativum, chromosomal proteins, cruciform DNA, high mobility group proteins.

\section{Introduction}

High mobility group (HMG) proteins are a ubiquitous class of non-histone chromosomal proteins whose biological role, despite intriguing speculations, is, so far, not yet elucidated. Since there is no biological assay for HMG function, these proteins are operationally defined as nuclear proteins which can be extracted from nuclei or purified chromatin by $0.35 \mathrm{M} \mathrm{NaCl}$ and are soluble in $2 \%$ TCA. Additional characteristics can be a high content of charged amino acids and a molecular weight lower than 30000 .

The HMG $1 / 2$ family (molecular weight of about 25000 ) is the most abundant HMG family. HMG 1 and 2 extracted from mammal tissues have a tripartite structure consisting of two internal repeats of a positively charged domain of about 80 residues and a polyacidic $C$ terminal domain. Each of the positively charged internal repeats have been called an $H M G$ box and represent the DNA binding motif of the protein.

Bianchi and collaborators (Bianchi, 1988; Bianchi et al., 1989, 1992) have shown that both the whole HMG 1 protein and HMG box 1 and 2, which have been cloned separately, interact specifically with four-way junction DNA, i.e. structures which are cross-shaped and contain angles of about $60^{\circ}$ and $120^{\circ}$ between their arms. Fourway junctions or 'cruciform DNA' arise in vivo as transient Hollyday junction during genetic recombination or from inverted repeat sequences under the effect of supercoiling. Both transcription and DNA replication, in which functions $H M G$ proteins are generally thought to be involved, can generate branched DNA molecules either directly or through the action of induced supercoiling (Bianchi et al., 1989; Ull et al., 1991). The binding to four-way junctions is structure-specific and sequence-independent, since HMG boxes can bind to several four-way junction DNA of unrelated sequences, but not to linear duplex or single strand DNA of the same sequences (Bianchi et al., 1992).

Studies on plant HMGs are more recent and far less numerous, but plant HMG-like proteins have been isolated and biochemically characterized from different sources (Spiker, 1984; Vincentz and Gigot, 1985; Moehs et al., 1988; Grasser et al., 1989, 1993; Ull et al., 1991). However, overall amino acid composition, peptide mapping, partial sequence analysis and immunological studies suggest a substantial difference between plant and animal HMG proteins. For instance, in contrast to the animal proteins which contain two HMG boxes, maize HMG 1 protein (whose amino acid sequence was deduced from the correspondent cloned cDNA insert) displays one such element alone, which is more similar ( $42 \%$ identity) to the animal HMG box 2 than to the amino-terminal

\footnotetext{
${ }^{3}$ To whom correspondence should be addressed. Fax: +39226604330 . 
HMG box 1 , while only a low similarity of the aminoterminal region of the maize $\mathrm{HMG}$ protein to $\mathrm{HMG}$ box 1 of the vertebrate proteins could be observed (Grasser and Feix, 1991). Despite little evidence of homology between animal and plant HMG proteins, the latter share the characteristics of vertebrate HMG proteins of being released from chromatin that has been treated with DNAase 1 and are found in chromatin fractions containing DNA complementary to mRNA (Spiker, 1988), both being properties related to structural proteins of transcriptionally poised chromatin.

Since the interaction with distorted DNA - preferentially they seem to recognize sharp angles-is the general property of HMG boxes, most probably central to their biological function, we decided to test whether plant nuclear proteins, operationally corresponding to HMG proteins, bind specifically to four-way junction DNA, like their animal counterpart. In this preliminary report we show that such proteins exist and, although their conclusive identification must be based on their amino-acidic sequence, their binding specificity to cruciform DNA could be used as an additional diagnostic criterion to assign them to the HMG class.

\section{Materials and methods}

\section{Plant material}

Pisum sativum cv. Lincoln was used for all the experiments. Seeds were treated for 10 min with $\mathrm{NaClO} 6 \%$ at $4{ }^{\circ} \mathrm{C}$, washed in cold water, and soaked at $2-4{ }^{\circ} \mathrm{C}$ for $16 \mathrm{~h}$. Embryos were manually removed and left in glycerol $30 \%$ at $-10^{\circ} \mathrm{C}$ until used.

\section{Isolation of nuclei}

A fraction of pure nuclei was obtained according to the procedure of Chiatante et al. (1990) partially modified to fit for a large quantity of starting material. The purity of nuclei was checked after staining with DAPI and direct observation at a fluorescence microscope (excitation filter BP 340-380, barrier filter LP 430) (data not shown).

\section{Isolation of HMG proteins}

Putative HMG proteins were extracted from gradient-purified nuclei with $0.35 \mathrm{M} \mathrm{NaCl}$ and $2 \%$ TCA, mostly according to one of the procedures suggested by Ull et al. (1991).

\section{Polyacrylamide gel electrophoresis}

Electrophoresis of proteins was performed in $15 \%$ polyacrylamide gels using the Tris/glycine/SDS system, according to Schagger and von Jagow (1987). The gels were then stained with Coomassie R-250.

\section{Construction of synthetic junction and linear DNA}

Oligonucleotides were synthesized by the phosphotriester method and purified by HPLC. Duplex and four-way junction DNA molecules were obtained by annealing the appropriate oligonucleotides and purified by electrophoresis. When desired one of the strands was labelled by treating with the Klenow fragment of DNA polymerase I and $\left(a{ }^{32} \mathrm{P}\right) \mathrm{dCTP}$. Details of the preparation and the characteristics of the synthetic strands have been described (Bianchi, 1988, 1992).

\section{Assay for DNA bınding to cruciform DNA}

For DNA binding experiments total extracts containing HMG proteins solubilized in non-denaturing medium, or single bands were used. In the latter case, the bands putatively corresponding to $\mathrm{HMG} 1$ and $\mathrm{HMG} 2$ proteins were visualized in SDS/ polyacrylamide gels by treatment with $1 \mathrm{M} \mathrm{KCl}$ for $30 \mathrm{~min}$, excised from the gel, homogenized in non-denaturating buffer at $60^{\circ} \mathrm{C}$ and renatured by gel filtration on $\mathrm{P} 6$ resin (Bianchi et al., 1989).

In band-shift experiments, total extracts or the recovered HMG 1-like and HMG 2-like proteins were added to labelled cruciform DNA $(1.5 \mathrm{nM})$ in DNA binding buffer ( $8 \%$ Ficoll, $200 \mathrm{mM} \mathrm{NaCl}, 10 \mathrm{mM} \mathrm{MgCl}_{2}, 10 \mathrm{mM}$ Hepes $\mathrm{pH} 7.9,5 \mathrm{mM}$ $\mathrm{KCl}, 1 \mathrm{mM}$ EDTA, $0.5 \mathrm{mM}$ DTT). After incubation for $10 \mathrm{~min}$ on ice, samples were applied to a 6.5 polyacrylamide gel in $0.5 \mathrm{M}$ TBE buffer and run for $4 \mathrm{~h}$ at $10 \mathrm{~V} \mathrm{~cm}^{-1}$ at room temperature. The gel was then dried, autoradiographed with Kodak X-AR film and exposed for $24 \mathrm{~h}$ at $-80^{\circ} \mathrm{C}$ with intensifying screen.

In competition experiments: (a) linear double strand DNA molecules of the same sequence as the oligonucleotides used in forming the cruciform structures, and (b) unlabelled cruciform DNA, were also added to the essay mixtures. The concentrations of competitors used are indicated in Fig. 4.

\section{Results and discussion}

When studying nuclear proteins a crucial step is to start from a highly purified fraction of nuclei. The fraction of nuclei we obtained was completely satisfying, so to rule out the possibility of contamination by cytoplasmic proteins. Yet, a strong contamination by an unknown nuclear protein could not be avoided (Fig. 1). According to most authors, at least one of the two contaminating bands should correspond to histone H1 (Moehs et al., 1988; Ull et al., 1991). Three weaker bands of molecular weight about $28000,24000,21000$, as estimated by standard molecular weight markers, were always found. We tried to prove that these three bands were indeed correspondent to vertebrate HMG proteins, but, unfortunately, antibod-

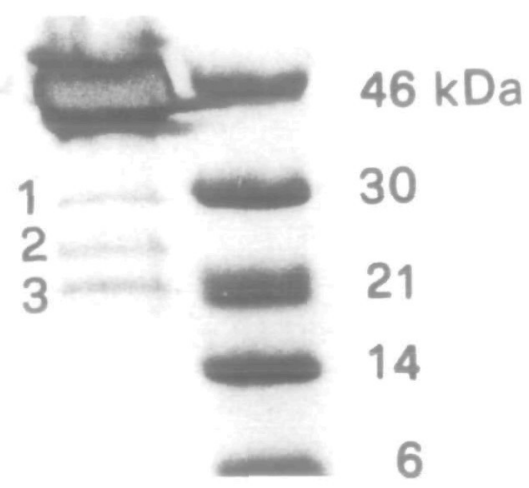

Fig. 1. SDS-PAGE of putative HMG proteins from pea embryos ( 1 . 2,3 in the left lane). The heavy band with lower mobility corresponds to the contaminating $\mathrm{H} 1$ histone. Right lane' molecular weight markers. 
ies against rat HMG 1 protein reacted non-specifically with the pea proteins; on the other hand, this is consistent with the low homology of plant HMG proteins to their animal counterpart. However, though our presumed HMG proteins have not been sequenced, their specific solubility in $0.35 \mathrm{M} \mathrm{NaCl}$ and $2 \%$ TCA and their molecular weight fit well with the putative $\mathrm{HMG}$ proteins isolated by Ull et al. (1991) from the same material. We believe that band 1 and 2 might correspond to animal HMG 1 and 2.

DNA binding assays were carried out using total extract containing HMGs resuspended in non-denaturing buffer; HMG 1-like and HMG 2-like bands excised from gel slices and renatured by gel filtration on P6 resin. Each of them was added to labelled cruciform DNA (Fig. 2) under the conditions described in Materials and methods. In all three cases the binding of the proteins caused a reduction of the electrophoretic mobility of the synthetic cruciform DNA, shown by a distinct mobility shift indicative of the formation of a protein/DNA complex (Fig. 3). The protein extracted from band 2 (lane 4 in Fig. 3) seemed to be less active and gave comparable but less clear-cut results. When other unknown nuclear proteins (bands excised from gels of total nuclear proteins) were tested at random in similar binding assays experi-

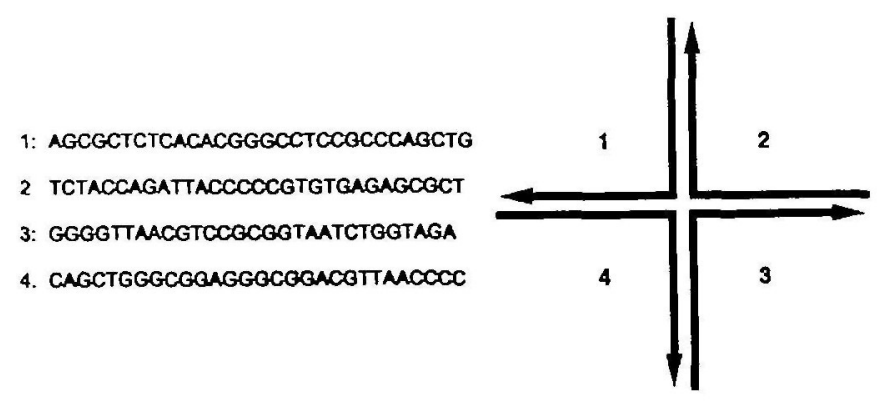

Fig. 2. Design of the synthetic cruciform DNA used.

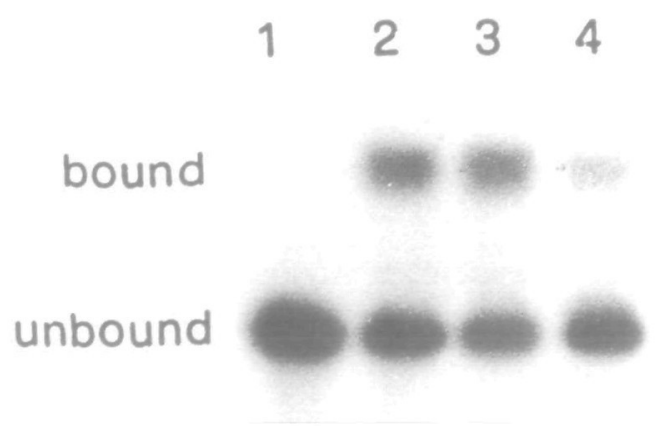

Fig. 3. Cruciform DNA-binding activity as revealed by gel-retardation assay. Reaction mixtures contained ${ }^{32}$ P-labelled cruciform DNA ( $1.5 \mathrm{nM}$ ) alone (lane 1), or added to total extract containing HMGs (lane 2 ), protein 1 (lane 3 ), protein 2 (lane 4 ) recovered from gel slice and renatured. The shift is evident for total HMGs (lane 2) and for HMG 1-like band (lane 3), less marked for HMG 2-like band (lane 4) ments, no shift of labelled cruciform DNA was ever observed (data not shown).

The cruciform-binding activity of pea HMG-like proteins is highly specific for DNA structure rather than for DNA sequence. This was shown in competition experiments where HMG 1-like protein (extracted and renatured from band 1) readily bound to the synthetic cruciform DNA, but did not bind to control linear DNA of the same sequence (Fig. 4A). Binding of HMG 1 was not even competed by a 1000 -fold higher concentration of linear DNA, but was competed, as expected, by cold four-way junction DNA (Fig. 4B). Quite similar results have been previously reported for animal $\mathrm{HMG}$ proteins (Bianchi et al., 1989; Bianchi, 1991).

There is increasing evidence that the consensus DNA structure for HMG 1 box binding is a local deformation of the DNA helix at the site of interaction. The interaction with distorted DNA, a general property of HMG boxes, must be crucial for their biological function. Deformations along the DNA molecule can be required for DNA transcription, replication, repair, and packaging. so that HMG may have a generalized role in DNA bending, looping, folding and wrapping, as proposed by Ferrari et al. (1992).

Moreover, evidence is emerging that HMG 1-related proteins can induce bends in linear DNA containing the central dinucleotides CA or TG (Landsmann and Bustin, 1993). So these proteins deform the DNA but also recognize bent DNA; as a matter of fact, the preferential binding to DNA in an already bent configuration might reflect a decreased energy requirement in the association step.

The present report, showing that HMG proteins from plants, like the animal ones, bind with high specificity to cruciform DNA is in agreement with the recent works of Kotani et al. (1993) reporting similar properties for an

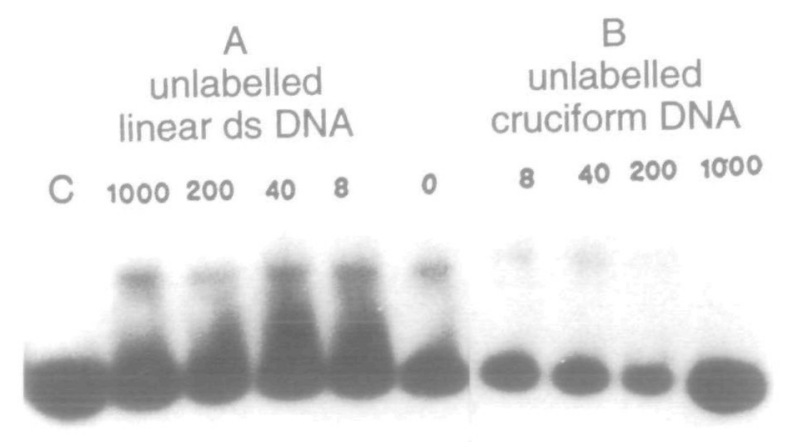

Fig. 4. Gel retardation assay to verify the binding specifity of HMGlike proteins to cruciform DNA. ${ }^{32}$ P-labelled cruciform DNA was incubated in the presence of pea HMG 1-like protein, and of nanomolar amounts $(1000,200,40,8,0)$ of unlabelled unspecific linear duplexes (A), and specific compettor (unlabelled cruciform DNA) (B) containing the same sequences. Extreme left lane: $\mathrm{C}=$ control (labelled cruciform DNA alone). 
HMG-like protein from Ustilago maydis and of Griess et al. (1993) about the interaction of maize HMG proteins with the rDNA external spacer region exhibiting DNA curvature. These findings, as a whole, add further evidence to the evolutionary conservation of the properties of HMG box all over the eukaryotic kingdoms, implying that it constitutes an essential module for the correct structure and/or function of DNA.

\section{References}

Bianchi ME. 1988. Interaction of a protein from rat liver nuclei with cruciform DNA. The EMBO Journal 7, 843-9.

Bianchi ME. 1991. Production of functional rat HMG 1 protein in Escherichia coli. Gene 104, 271-4.

Bianchi ME, Beltrame M, Paonessa G. 1989. Specific recognition of cruciform DNA by nuclear protein HMG 1. Science 243, 1056-9.

Bianchi ME, Falciola L, Ferrari S, Lilley DMJ. 1992. The DNA binding site of HMG 1 protein is composed of two similar segments (HMG boxes), both of which have counterparts in other eukaryotic regulatory proteins. The EMBO Journal 11, 1055-63.

Chiatante D, Brusa P, Levi M, Sgorbati S, Sparvoli E. 1990. A simple protocol to purify fresh nuclei from milligram amounts of meristematic pea root tissue for biochemical and flow cytometry applications. Physiologia Plantarum 78, 501-6.

Ferrari S, Harley VR, Pontiggia A, Goodfellow PN, LovellBadge R, Bianchi ME. 1992. SRY, like HMG 1, recognizes sharp angles in DNA. The EMBO Journal 11, 4497-506.

Grasser KD, Feix G. 1991. Isolation and characterization of maize cDNAs encoding a high mobility group protein dispalying a HMG-box. Nucleic Acid Research 19, 2573-7.
Grasser KD, Maier VG, Feix G. 1989. A nuclear casein II kinase from maize endosperm phosphorylating HMG proteins. Biochemical and Biophysical Research Communication $162,456-63$.

Grasser KD, Wohlfarth T, Baumlein H, Feix G. 1993. Comparative analysis of chromosomal HMG proteins from monocotyledons and dicotyledons. Plant Molecular Biology 23, 619-25.

Griess EA, Grasser KD, Feix G. 1993. Repeat units from a maize rDNA external spacer region exhibit DNA curvature and interact with high mobility group protenns. Planta $191,524-31$.

Kotani H, Kmiec EB, Holloman WK. 1993. Purification and properties of a cruciform DNA binding protein from Ustilago maydis. Chromosoma 102, 348-53.

Landsman D, Bustin M. 1993. A signature for the HMG 1 box DNA binding proteins. Bio Assays 15, 539-46.

Moehs CP, McElwain F, Spiker S. 1988. Chromosomal proteins of Arabidopsis thaliana. Plant Molecular Biology 11, 507-15.

Schagger H, von Jagow G. 1987. Tricine sodium dodecyl sulphate polyacrylamide gel electrophoresis for the separation of proteins in range from 1 to $100 \mathrm{kDa}$. Analytical Biochemistry 166, 368-79.

Spiker S. 1984. High mobility group chromosomal proteins of wheat. Journal of Biological Chemistry 259, 12007-13.

Spiker S. 1988. Histone variants and high mobility group nonhistone chromosomal proteins of higher plants: Their potential for forming a chromatin structure that is either poised for transcription or transcriptionally inert. Physiologia Plantarum 75, 200-13.

UII MA, Herrero ME, Franco L. 1991. Putative high mobility group non-histone chromosomal proteins from pea (Pisum sativum). Plant Science 75, 55-62.

Vincentz M, Gigot G. 1985. HMG-like proteins in barley and corn nuclei. Plant Molecular Biology 4, 161-8. 